\title{
NG ORK \\ H \\ The Impact of Obesity on Adult Mortality: Assessment of Estimates with Applications
}

\author{
Alberto Palloni \\ Hiram Beltrán-Sánchez
}

Working Paper

2017-044

05/201, Revised 6/2017

HUMAN CAPITAL AND

ECONOMIC OPPORTUNITY

GLOBAL WORKING GROUP
The University of Chicago

1126 E. 59th Street Box 107

Chicago IL 60637 


\title{
The Impact of Obesity on Adult Mortality: Assessment of Estimates with Applications
}

\author{
Alberto Palloni \\ Labor and Population Unit, RAND Corporation \\ Center for Demography and Health of Aging, University of Wisconsin-Madison. ${ }^{1}$ \\ Hiram Beltrán-Sánchez \\ Department of Community Health Sciences \\ Fielding School of Public Health \& California Center for Population Research, UCLA. ${ }^{2}$
}

\footnotetext{
${ }^{1}$ Palloni's research was supported by the National Institute on Aging via research project grants R01AG016209, R03-AG015673, R01-AG018016, and R37-AG025216), by a Fogarty International Center award for Global Research Training in Population Health, D43-TW001586, and by core grants to the Center for Demography and Ecology, R24-HD047873, and to the Center for Demography of Health and Aging,P30AG017266), at the University of Wisconsin-Madison

${ }^{2}$ Beltrán-Sánchez's research was supported by a grant R24 HD041022 from the National Institute of Child Health and Human Development to the California Center for Population Research at UCLA.
} 


\begin{abstract}
In this paper we assess properties of commonly used estimates of total effects of obesity on mortality and identify consequences of these properties for inferences. We argue that standard estimates have important shortcomings that at best limit the reach of inferences and at worst lead to misleading conclusions. Although some of these limitations are routinely acknowledged, rarely is their use accompanied by careful scrutiny of their weaknesses, let alone by a quantitative assessment of their sensitivity to violations of some stringent assumptions on which they are based. In this paper we develop an integrated framework based on a multistate hazard model to describe properties of the simpler standard estimates, identify conditions under which their performance is best, and define the nature of biases and interpretational ambiguities that emerge when empirical conditions depart more than modestly from optimal ones. In particular, we show formally that estimates from limited panel data and two-state hazard models with obesity as a covariate, the workhorse in this area, produce estimates that are difficult to interpret and compare across studies and, in some cases, biased. Finally, we propose a simple procedure that can be employed when the use of conventional two-state models is risky and illustrate its application to an a empirical case.
\end{abstract}

Key Words: Obesity, T2D, mortality, multistate hazard model, two-state hazard model 


\section{Introduction}

In the last ten years adult populations everywhere have experienced rapid increases in body weight ( $\mathrm{Ng}$ et al. 2014). The problem is particularly salient in low to middle income countries in Latin America, Asia and Africa where the increase is faster and its consequences more detrimental. For example, in 2012 the prevalence of adult obesity in Mexico was of the order of 33 percent (Cecchini and Sassi 2014; Ng et al. 2014; FAO 2015) placing the country in the first quartile of the obesity distribution in South, Central and North America. Other countries in these regions are rapidly approaching similar levels. Furthermore, because child obesity also has swiftly increased everywhere (Ng et al. 2014) and is a likely precursor of adult obesity (Brisbois et al. 2012), future increases in adult obesity prevalence are very likely to continue apace (Biro and Wien 2010).

There is ample evidence showing that obesity, but less so overweight, are closely linked to health risk factors such as insulin resistance, Type 2 diabetes (T2D), dyslipidemia, coronary heart disease, sleep apnea, cognitive dysfunction, some cancers, and liver and kidney disease (Guh et al. 2009; Ix and Sharma 2010). And while the impact on chronic conditions and disability on mortality is not questioned, there is still controversy about the precise magnitude and even direction of the total effect of obesity on mortality. Indeed, some research suggests deleterious effects, some show a neutral impact and, in some cases, there is evidence of a beneficial influence, albeit at older ages. A very recent meta-analysis of more than one hundred studies found that normal and overweight individuals share similar mortality risks and that excess mortality is concentrated among those classified as obese or morbid obese (grade 2 and 3) (Flegal et al. 2013). This approximately J-shaped function (or U-shaped if one includes underweight individuals) of the obesity-related mortality risk has been confirmed by some studies (Al Snih et al. 2007; Fontaine et al. 2003) but not in others (Preston and Stokes 2011; Preston et al. 2014).

Weak concordance of empirical estimates of mortality effects of obesity poses a problem for projections and forecasts of the health damage that past, current and future trends of obesity imply, is an obstacle for transparent inferences from comparative studies, and could jeopardize the design of informed health policy interventions.

There are many reasons why empirical findings may produce inconsistent results. Some are conceptual and related to the definition of obesity and overweight (selection of indicators and metric), others are formal and involve specification of the functional form of relations of interest (continuous, discrete, thresholds). In this paper we are concerned with properties of estimates most commonly employed in empirical research and on the consequences of these properties for inferences. We argue that the most commonly used estimates have important shortcomings. While some of these shortcomings are explicitly recognized by researchers in the field, we know of no integrated framework to assess biases or to identify conditions that influence the reach of inferences from alternative models. In particular, we show formally that estimates from limited panel data using standard two-state hazard models with obesity as a covariate, the workhorse in this area, produce estimates that can be difficult to interpret and/or compare across studies and, in some cases at least, are biased. Our aim is to identify with precision the sources of difficulties, to define under what conditions inferences may be damaged and, lastly, to describe a procedure that attenuates the magnitude of the damage. The ideal strategy to remove these shortcomings is to estimate full multistate hazard models but this is unfeasible with limited panels, the most widely available source of information in this area of research. To circumvent the need for estimates from multistate hazard models we propose a simple procedure to compute substitutes and illustrate with an application to an empirical case. Although the procedure is not problem-free it does produce estimates with 
transparent interpretation that can be compared across populations, and are suitable inputs in projections and forecasts.

\section{Conventional estimates of mortality effects of obesity}

In this section we develop expressions for estimates of effects of obesity on mortality under scenarios commonly encountered in empirical research. In a large number of cases the investigator can avail herself of data originating in limited panels. These are panel studies consisting of two or more (frequently only two) waves, with irregular and oftentimes lengthy interwave periods, and limited or no recall of retrospective events. To provide background and motivation we start with a simple illustration of a commonly employed strategy whereby the investigator estimates a two-state hazard model with no control for antecedent illnesses, the preferred approach in this field. We show first informally, and then more formally with a multistate hazard representation, that the estimates produced by this approach are always difficult to interpret and in some cases biased.

\subsection{Definition of the target parameter}

Before discussing the nature of estimates it is useful to define the population parameter that most empirical studies seek to estimate. By and large, the target parameter is the 'total' effect of obesity on relative risks of adult mortality. As in standard path analysis, a total effect is the sum of the contribution of all direct and mediated paths conducing from an origin variable or state to an outcome. Figure 1 is a simple representation that includes $k=4$ mediating obesity-related illnesses. There are $(k+1)$ paths linking the condition of interest (obesity) and the outcome (mortality), $k=4$ of them require the mediation of an illnesses and one is direct. One may question whether obesity has any direct effect at all on mortality and, if so, the 'direct' path could stand for effects of obesity through illnesses that are unknown or unmeasured. In a path analysis we measure the 'strength' of each path using a metric of path coefficients, namely, $\beta_{O 2}, \beta_{O 3}, \ldots, \beta_{O k}$ to reflect paths from obesity to illness (with $\beta_{O D}$ reflecting the 'direct' effect) and $\gamma_{1 D}, \ldots, \gamma_{k O}$ to reflect the paths from illnesses to mortality. The total association between obesity and mortality is estimated using the quantity $\tau=\sum_{j=1}^{k} \beta_{O j} \gamma_{j D}+\beta_{O D}$. We show below that, when the process is represented by a multistate hazard model, the algebra of effects of obesity on mortality is analogous to that of path analysis. The only difference is that instead of path coefficients the metric consists of instantaneous transition rates. ${ }^{1}$

\subsection{Standard two-state model with no control for illness}

The mainstay strategy to obtain the total effect of obesity on mortality consists of estimating effects of a dummy variable for obesity (obesity and/or overweight) with no controls for antecedent illness but including other controls (age, gender, etc.,...). These models are generally estimated from panel data that retrieve information about events in the interwave period from retrospective recall but only for individuals who survive across waves. ${ }^{2}$ Interwave events and their timing to decedents are rarely, if ever, identified. Assume the force of mortality of obese individuals is $\mu=\mu_{o} \exp (\rho)$, where $\rho$ is the 'effect' of obesity and $\mu_{o}$ is the force of mortality in the normal weight population. ${ }^{3}$ If

\footnotetext{
${ }^{1}$ A caveat regarding terminology. Unless explicitly noted we use the expressions 'obese sub-population' or 'obese individuals' to refer to those whose BMI exceeds 25, thus including both overweight and obese individuals. When needed, we specialize the term obese to those whose BMI exceeds 29.

${ }^{2}$ Even though the panel may consist on more than two waves, we will speak throughout of first and second wave of a panel to mean an initial and the immediately following wave.

${ }^{3}$ As written, the model is agnostic about mechanisms. Obesity will trigger illnesses that lead to excess mortality and, in addition, obesity itself may cause physiological damage that increase excess mortality risks. Thus, the
} 
all transitions occur at the end of the exposure period the log likelihood function of the observed sample is:

$$
\ln (L)=-\left[N_{0}+N_{1} \exp (\rho)\right] \mu_{0}+\left(D_{0}+D_{1}\right) \ln \left(\mu_{0}\right)+D_{1} \rho .
$$

In this expression the quantities $D_{0}$ and $D_{1}$ are death counts in the obese and normal weight sub-populations and $N_{0}$ and $N_{1}$ are the total exposure contributed by each of them. The classic proportional hazard estimators are $\hat{\mu}_{0}=D_{0} / N_{0}$ and $\exp (\hat{\rho})=\left(D_{1} / N_{1}\right) /\left(D_{0} / N_{0}\right)$ for the relative risk. These estimates are unbiased and consistent under standard assumptions about independent censoring and proportionality of effects. The model, however, will produce inappropriate or ambiguous results with limited panel data in three situations:

1. Preceding debilitation: Assume that, unbeknownst to the investigator, a fraction $f$ of individuals who are normal weight in the first wave did experience an obesity-related illness that led to debilitation manifested in weight loss prior to the first wave. Let their mortality risk be $\mu^{\prime}=\exp (\delta+\rho)$. The maximum-likelihood (ML) estimator of obesity relative risk defined above will be an estimate of $\exp (\rho) /((1-f)+f \exp (\delta))$ rather than of $\exp (\rho)$. If the target parameter is $\rho$, the empirical estimate will have a proportional downward bias equal to $(1 /((1-f)+f \exp (\delta)))$. Note that if $\delta>=0.50$, a small value of $f=0.10$ is sufficient to produce a bias exceeding 10 percent. An illustration of this type of mechanism involves T2D. Obesity is thought to be causally related to the incidence of T2D and, in addition, the onset of this illness oftentimes precipitates a period of sudden weight loss. It is possible that some individuals who are below a BMI threshold at the time of the first wave may have been obese in a period preceding the first wave, contracted T2D as a result of obesity, and are then exposed to higher mortality risks than normal weight individuals in the interwave period under investigation.

2. In-panel debilitation: Assume $g$ and $h$ are fractions of the obese and normal weight subpopulations who, at the time of the first wave, already have or develop a chronic illness unrelated to obesity and die as a consequence of it before the subsequent wave. ${ }^{4}$ To abbreviate we will refer to such illnesses as 'ancillary', to mean they are not causally related to obesity but could be spuriously associated with it. The ML estimate of the relative risk of obesity will be $\exp (\rho)[((1-g)+g \exp (\nu)) /((1-h)+h \exp (\eta))$, where $\exp (\nu)$ and $\exp (\eta)$ are the relative risks of death associated with the ancillary disease in the obese and normal weight sub-populations respectively. If the prevalence of the ancillary chronic illness and the excess mortality associated with it do not differ by obesity status, the ML estimate will be a consistent estimate of the target parameter. Otherwise the estimate will be biased. Two situations can generate biases in the the conventional two-state estimate: (a) when the ancillary illness has different mortality risks in the obese and normal weight sub-populations but differences are not directly related to obesity; ${ }^{5}$ or (b) when there is sample selection that dampens the relative mortality risk of the illness over time and does so differentially in the obese and non

parameter $\rho$ captures total effects of obesity, including effects on illnesses, direct effects of illnesses on mortality and, if there are any, direct effects of obesity on mortality. Its analogue in a path model is the association between the dependent and independent variable, $\tau$.

${ }^{4}$ Because the investigator does not control for illness in the model, the distinction between preexistence (prevalence of) the disease at the time of the first wave and incident cases during the interwave is irrelevant. The distinction matters when the investigator controls for illness because then incident cases that result in death before the second wave will go unnoticed.

${ }^{5}$ For instance, when there are omitted variables that influence both obesity and the ancillary illness mortality risks 
obese sub-populations. ${ }^{6}$ We show below that the direction of biases depends on the exact magnitude of the parameters involved. Note also that comparison of estimates across two different populations with different values of $g, h, \nu$ or $\eta$ but identical $\rho$, will lead to ambiguous interpretations. An illustration of this type of mechanism involves smoking and lung related diseases. If their prevalence or relative mortality risks are higher among the obese population (but obesity has no influence on smoking or smoking mortality risks), we will attribute the excess deaths to obesity. If the association is causal and, for example, obesity increases smoking propensities or smoking related mortality risks, the attribution captures one mechanism through which obesity indeed contributes to excess mortality. But if the association is due to dependence on common causes or antecedent selection the attribution is misleading.

3. Preceding and in-panel debilitation: when the illness producing preceding and in-panel debilitation are the same, the ML estimate of $\exp (\rho)$ will include the ratio of the two proportional biases defined above. Thus, the magnitude and direction of the net bias will depend on the relative size of each component bias.

The foregoing is an informal rendition of the problems generated by limited panels. All three situations are admitted as possible in the literature and most researchers issue warnings about their impact. However, these warning are rarely followed by a rigorous empirical assessment of the problem they may cause. In the section below we discuss a more formal derivation of the functional form of target estimates under conditions dominated by mechanisms (1) to (3), show the pathways of influence, and describe conditions under which they become a threat to inferences.

\subsection{Multistate representation}

Assume that a multistate model generates all the data and that the observables originate in a detailed longitudinal follow-up study. Individuals can occupy one of 7 states and experience a total of 10 possible transitions. Figure 2 is a graphic representation of states and permissible transitions. We use alphabetic character to label states: $N O_{H}$ and $O_{H}$ are for normal weight and obese healthy states, $N O_{U_{A}}$ and $O_{U_{A}}$ are reserved for states occupied by non-obese and obese unhealthy due to ancillary conditions and, finally, $N O_{U_{O}}$ and $O_{U_{O}}$ are states occupied by non-obese and obese individuals who are unhealthy due to chronic illnesses related to obesity. If a normal weight individual contracts T2D she is classified in state $N O_{U_{O}}$ whereas an obese individual who contracts lung cancer is classified in state $O_{U_{A}}$. As is standard practice, all transition rates are denoted by $\mu_{i j}$, where $i$ are subscripts denoting a state of origin $i$ and a state of destination $j$. Transitions from $O_{U_{O}}$ (state 6) to $N O_{U_{O}}$ (state 4) and from $O_{U_{A}}$ (state 5) to $N U_{U_{A}}$ (state 3) are events defined by weight loss due to an obesity-related and ancillary illness respectively. In a world where proportionality is

\footnotetext{
${ }^{6}$ Any selective attrition that produces different composition by frailty of survivors in the obese and non-obese sub-populations at the time of onset of exposure can result in differential mortality due to the ancillary illness
} 
universal the complete model is defined by the following equations

$$
\left.\begin{array}{rr}
\mu_{13}= & \mu_{13} \\
\mu_{14}= & \mu_{14} \\
\mu_{25}= & \mu_{13} \exp (\lambda) \\
\mu_{26}= & \mu_{14} \exp (\delta) \\
\mu_{37}= & \mu_{37} \\
\mu_{47}= & \mu_{47} \\
\mu_{57}= & \mu_{37} \exp (\phi) \\
\mu_{67}= & \mu_{47} \exp (\beta) \\
\mu_{53}= & \mu_{53} \\
\mu_{64}= & \mu_{64} \\
\hat{\mu}_{37}= & \mu_{37} \exp (\alpha) \\
\hat{\mu}_{47}= & \mu_{47} \exp (\varphi)
\end{array}\right\}
$$

The parameters $\lambda$ and $\delta$ control the risks of contracting obesity-related and ancillary illnesses in the obese population; $\phi$ and $\beta$ are measures of excess mortality due to obese-related and ancillary causes in the obese population. The transition rates $\mu_{53}$ and $\mu_{64}$ represent risks of weight loss experienced by those initially obese and as a result of an ancillary and an obesity-related illness respectively. These individuals may experience different mortality risks $\left(\hat{\mu}_{37}\right.$ and $\hat{\mu}_{47}$ ) than those who occupy states 3 or 4 but originate in state 1 . To simplify, and because their consideration does not alter the expressions derived below, we ignore the role played by covariates such as gender, ethnicity, age, and assume that all inferences are conditional on these covariates.

It bears noting that even though this model is more complete than conventional two-state models, it still is quite a blunt tool to represent complex relations involving obesity, illness, and mortality. Thus, for example, while the transition from state 2 to state 6 implies physiological damage that depends on the illness or condition, we only assume the existence of a single (average) excess risk associated with it. Similarly, all potential protective effects of obesity that could occur because of differential composition by frailty of individuals occupying states 2 and 5 , on one hand, and state 6 , on the other, are omitted. Thus, while the multistate model is less simplistic than the two-state model, it is not complex enough to be a complete structural model.

We now derive expressions for two-state hazard model estimates from limited panels when observations are generated by the multistate model. These expressions are the 'reduced' forms of the structural representation of the multistate model and will depend on combinations of transition rates embedded in it.

\subsection{Conventional estimation with a two-state model and limited panels}

Assume the investigator has access to data from two waves and incomplete recall information so that she cannot identify key events that took place in the interwave period to individuals lost to interview nor identify others that may have occurred shortly before the first wave. In particular, changes in body weight or illnesses experiences of individuals who are censored or who die between waves, will not be recorded accurately or at all. We begin with the conventional two-state model, with no control for either obesity-related or ancillary illnesses. This is the preferred tool in most 
studies of the relation between obesity and mortality. ${ }^{7}$ We will assume throughout that relevant transitions take place in the middle of observation period, irrespective of state of origin. A typical two-state model for observations between two panels is as follows:

$$
\mu=\mu_{0} \exp (\rho Z)
$$

where $\mathrm{Z}$ is a single, $1 / 0$ binary, covariate for obesity status and $\rho$ is the target parameter. The two-states ML estimates of $\mu_{0}$ and $\rho$ are $\hat{\mu}_{0}=D_{0} / N_{0}$ and $\exp (\hat{\rho})=\left(D_{1} / N_{1}\right) /\left(D_{0} / N_{0}\right)$, respectively, and $\rho$, the target parameter, is for the total effect of obesity on mortality. Because the multistate model generates the observations, we can express the two state model estimates as a function of parameters in the multistate model. We consider two cases, the first when there is no preceding debilitation $\left(\mu_{53}=\mu_{64}=0\right)$ and the second when all transitions are possible during the interwave period.

\subsubsection{Case I: in-panel debilitation only $\left(\mu_{53}=\mu_{64}=0\right)$}

The two-state estimate of the relative risk due to obesity is given by ${ }^{8}$

$$
\exp \left(\hat{\rho}_{I}\right)=\exp (\lambda+\phi) w+\exp (\delta+\beta)(1-w)
$$

where $w=\mu_{13} \mu_{37} /\left(\mu_{13} \mu_{37}+\mu_{14} \mu_{47}\right)$. The weight $w$ represents the relative strength of one of the two paths to reach state 7 from state 1 (or 2), the other being through a sojourn in state 4 (or 6 ). Correspondingly, the term $\exp (\lambda+\phi)$ is the relative risk in the obese population of transitioning from state 2 to state 7 or, alternatively, the total effect of obesity on mortality due to ancillary illness. Analogously, $\exp (\delta+\beta)$ is the total effect of obesity on mortality due to obesity-related illnesses.

The target of investigation is the total effect of obesity which, in the multistate rendition, corresponds to the strength of the transition rate from state 2 to 7 with a sojourn in state 6 or, equivalently, the quantity $\exp (\delta+\beta) .{ }^{9}$ However, the two-state estimate also reflects excess risk of contraction of and relative risk of mortality due to normal weight-related illnesses, e.g. the quantity $\exp (\lambda+\phi)$. Thus, if $(\lambda+\phi)>0$ the two-state estimate will overestimate the target parameter.

It is only in two extremes cases that the two-state estimate is concordant with conventional interpretations, e.g. a measure of the total effect of obesity or the total sum of direct effects and those due to obesity-related illnesses in the obese sub-population. The first case is when the quantity $\mu_{13} \mu_{37}$, a measure of relative strength of the path to state 7 via ancillary illnesses, is either small or, equivalently, when either incidence or lethality of the ancillary illness is 0 . If the interwave period is short relative to the magnitude of the risks, in-panel debilitation will be rare and should cause no damage to estimates. The second case is when $(\lambda+\phi)=(\delta+\beta)$, that is, when the gross effect of obesity on mortality are identical irrespective of the nature of illness. In all other cases the

\footnotetext{
${ }^{7}$ Although the exact model specification may include other controls, we ignore them in our derivations since they do not affect interpretations.

${ }^{8}$ In what follows we use approximations to exact expressions derived in section 1 of the Appendix.

${ }^{9}$ It should be kept in mind that a two-state model with no control for preceding illness cannot distinguish between cases of illnesses that exist at the time of the first wave and new cases verified during the interwave period. If so, it does not matter whether deaths are associated with prevalence of the illness at the time of the first wave or with new cases. The presence of the parameter $\delta$ in the case of obesity-related illnesses (and $\lambda$ in the case of ancillary illnesses) is simply a result of the assumption made in the multistate representation that all deaths in the interwave period are associated with new cases. Alternatively, one could assume that $\delta$ (or $\lambda$ ) is equal to zero but then introduce the prevalence of the disease at the time of the first wave. The formal result is equivalent but will be reflected in a measure of prevalence instead of an incidence rate.
} 
two state estimate will confound the impact of obesity-related and ancillary illnesses and could be downwardly biased. The bias will be smallest in the apparently harmless case when $(\lambda+\phi)=0 .{ }^{10}$ Reduction of either $\lambda$ or $\phi$ can take place as a result of sample selection: if high values of $\mu_{26}$ leave sturdier individuals in state 2 who then go on to experience lower risks $\mu_{25}$ and $\mu_{57}$, the value of $(\lambda+\phi)$ will be driven to 0 . If selection via $\mu_{26}$ is extreme relative to selection via $\mu_{13}$, the values of $\phi$ and $\lambda$ can become negative and $\exp (\tilde{\rho})$ will contain a substantial downward bias. This artifact could partially explain the beneficial effects of obesity at older ages reported in some empirical research (Al Snih et al. 2007).

These results suggest that inferences from comparisons of two-state estimates in different samples, at different times and in different populations should be used with caution because the data being compared might be produced by different structural parameters. Similar values of the total effect of obesity from a two-state model with no control for ancillary illness may result from different processes if populations differ in terms of $\beta$ or $\delta$. Two populations characterized by identical total effects of obesity on mortality could differ on the obesity-related incidence and lethality of T2D. These differences will be masked by ancillary illnesses acting through $\lambda$ or $\phi$. As a consequence of these features, results of meta-analyses of effects of obesity on mortality should be interpreted with a great deal of caution when researchers do not control or account for potential heterogeneity in the relations identified here.

\subsubsection{Case II: in-panel and preceding debilitation $\left(\mu_{53}\right.$ and $\left.\mu_{64} \neq 0\right)$}

Suppose that loss of body mass results from either an obesity-related or ancillary illnesses and that individuals who experience it also experience different (possible higher) mortality risks. The corresponding transitions are not observed by the investigator at the time of the first wave. The two-state estimate with no control for illness will be

$$
\exp \left(\hat{\rho}_{I I}\right)=\exp \left(\hat{\rho}_{I}\right) /(1+\Gamma)
$$

where $\Gamma \sim\left(\exp (\lambda+\alpha) \mu_{13} \mu_{53} \mu_{37}+\exp (\delta+\varphi) \mu_{14} \mu_{64} \mu_{47}\right)$ and $\Gamma>0$ is the magnitude of the contribution of preceding debilitation and will always induce a downward bias. The magnitude of this bias will be a function of the strength of the two paths leading from state 2 to state 7 with intermediate sojourns in states 3 and 4.These two paths are captured by the expressions $\mu_{25} \mu_{53} \hat{\mu}_{37}=\mu_{13} \mu_{53} \mu_{37} \exp (\lambda+\alpha)$ and $\mu_{26} \mu_{64} \hat{\mu}_{47}=\mu_{14} \mu_{64} \mu_{47} \exp (\delta+\varphi)$. The magnitude of $\Gamma$ will be driven to zero when the size of the risks is too small relative to the time interval in which the transitions must occur. This is more likely to happen in all waves, except the first one, when interwave periods are short relative to the magnitude of the transition rates involved. The magnitude of the bias will be higher in the first wave.

\section{Alternative procedures}

The problems identified above emerge due to two different mechanisms. In-panel debilitation is analogous to the existence of omitted covariates and the distortions caused by it can be dealt with standard multivariate approaches. However, this strategy has been discouraged among those whose goal is to obtain a total effects of obesity on mortality. Preceding debilitation is akin to reverse causality and the ensuing bias cannot be eliminated unless the underlying model reflects the causal mechanism embedded in the multistate hazard model. In sections (3.1-3.3) we discuss procedures that partially resolve these problems.

\footnotetext{
${ }^{10}$ This is because when $\exp (\delta+\beta)>1$ then $(w+\exp (\delta+\beta)(1-w))<\exp (\delta+\beta)$.
} 


\subsection{Two-state model with control for ancillary illness}

If the investigator knows about the presence of ancillary illnesses (from previous waves, decedents' relatives reports, or available health records), a two state model that includes a control for the target ancillary illness will reduce biases and ambiguities. The model is as follow

$$
\mu_{i}=\mu_{0} \exp \left(\beta Z_{i}+\kappa I_{i}+\lambda Z_{i} I_{i}\right)
$$

where $\mathrm{Z}$ is $1 / 0$ binary variable for obesity and $\mathrm{I}$ is $1 / 0$ binary variable for presence of the target condition and $i=0,1,2,3$ represent the following sub-populations: normal weight and 'healthy' (0), normal weight with the condition (1), obese and 'healthy' (2) and obese and with the condition (group 3). This model includes explicitly only the target illness causing in-panel debilitation and assumes there are no other illnesses that, unbeknownst to the investigator, operate as the target illness does. Thus, 'healthy' and 'ill' are defined only in reference to the target illness alone. Under these conditions, the parameter $\beta$ is the target parameter and reflects the total effects of obesity, e.g. effects working through obesity related illnesses and any direct effect.

After writing down the log likelihood function, taking derivatives relative to $\hat{\mu}_{0}$ and setting it to 0 we get:

$$
\hat{\mu}_{0}=\sum_{i=0}^{i=3} D_{i} \div\left(N_{0}+N_{1} \exp (\hat{\kappa})+N_{2} \exp (\hat{\beta})+N_{3} \exp (\hat{\lambda})\right)
$$

The following are trial solutions for the estimates of the other parameters:

$$
\left.\begin{array}{l}
\kappa^{\prime}=\exp (\hat{\kappa})=\left(D_{1} / N_{1}\right) / \mu_{0} \\
\beta^{\prime}=\exp (\hat{\beta})=\left(D_{2} / N_{2}\right) / \mu_{0} \\
\lambda^{\prime}=\exp (\hat{\lambda})=\left(D_{3} / N_{3}\right) / \mu_{0}
\end{array}\right\}
$$

where $D_{i}$ is the number of deaths in group $i$ and $N_{i}$ is the exposure in group $i$. The last three expressions are the ML estimates of relative risks if and only if $\hat{\mu}_{0}=D_{0} / N_{0}$. Since the system of equations for partial derivatives of the likelihood function has only one solution, the above expressions must define the ML estimates. Parameter $\kappa^{\prime}$ is a measure of the relative risks of mortality due to the ancillary illness in the normal weight population. $\beta^{\prime}$ is a relative risk reflecting the total effect of obesity on mortality working through illnesses other than the ancillary one. $\lambda^{\prime}$ is a measure of excess mortality among obese individuals who experience the target disease. It reflects the combined effects of obesity via the ancillary illness and other chronic conditions. If the effects of obesity and the ancillary condition are additive we could write $\hat{\lambda}^{\prime}=\exp (\hat{\kappa}+\hat{\beta})$. Otherwise, $\hat{\lambda}^{\prime}=\exp (\hat{\gamma}+\hat{\beta})$ with $\hat{\gamma} \neq \hat{\kappa}$. If one lets the number of obese and normal weight individuals in the sample be the same in each group (but not necessarily the prevalence of the target illness), we can express the estimate from the two-state model with no control for ancillary illness as a function of the estimate of the two-state model with a control for illness:

$$
\exp (\tilde{\rho})=\exp (\hat{\beta})((1-f)+f \exp (\hat{\gamma})) /((1-g)+g \exp (\hat{\kappa}))
$$

where $\tilde{\rho}$ is the estimate from the two state model with no controls and $f$ and $g$ are the prevalence of the chronic illness among those who are obese and normal weight respectively. This expression shows the contribution of both prevalence and excess mortality of the target disease to the difference between the two estimates. 
Two remarks. First, the strategy above solves the in-panel debilitation problem only if the latter is produced entirely or mostly by prevalence of ancillary illnesses whose time of onset precedes the first wave. The control cannot reduce biases produced by debilitation associated with interwave incidence cases. Second, it may be possible to control for 'proxies' of illnesses contracted before the first wave or during the interwave period. For example, one could include a control for smoking to avoid distortions produced by ancillary illnesses potentially associated with smoking. This is frequently done in most studies. But it only targets smoking-related conditions and is, therefore, an incomplete control.

In summary, estimates of obesity relative mortality risks from a two-state hazard models with no control for antecedent illness can oftentimes lead to biases and/or misleading interpretation of effects. When the mechanism creating the problem is interwave debilitation a model with a control for the target illness (or illnesses) is sufficient to adjust for biases or to fine-tune interpretations. This suggests that the choice between a two-state model with or without a control for illness should not follow the default with no control, as is conventionally done, but must depend on a careful assessment of conditions that produce (inhibit) in-panel debilitation.

\subsection{Two-state model with control for antecedent weight loss and cause of death}

Preceding debilitation is a much tougher problem to solve When there is preceding debilitation it is not possible to retrieve an unbiased estimate of the total effects of obesity without directly estimating a multistate hazard model. Simple solutions, such as the one suggested above for the case of in-panel debilitation are not feasible either. However, additional information on extreme weight loss before the first wave is potentially useful to produce an alternative estimate. In fact, suppose the investigator elicits information on weight loss during a short period of time preceding the first wave. This information can originate in retrospective reports from the decedent's relatives or other informants. A modified two-state model that includes a 1/0 dummy variable to reflect the existence of reported extreme weight loss can then be estimated. It is easy to show that the estimate from this two-state model variant will overestimate the target parameter. If so, the true value of the parameter must be within a range defined by the naive two-state estimate (underestimate) and by the two-state variant (overestimate). When the range is small the investigator can be assured that in-panel debilitation is unlikely to be a source of bias.

\subsection{A three stage procedure}

An alternative strategy that addresses simultaneously in-panel and preceding debilitation consists of employing a three-stage procedure. In a first stage one estimates the effects of obesity on the incidence of obesity-related illnesses. The second stage estimates the mortality risks of the obesityrelated illness in the obese population. Finally, the third stage blends the estimates from the first two stages into a single estimate of the total effect of obesity on mortality. To simplify, we consider only one major obesity related illness, T2D, but the procedure can be generalized to include others.

\subsubsection{Stage 1: two state model for the effect of obesity on T2D}

We assume that the investigator has reliable assessments of T2D either via self reports or standard blood tests and, using the population of diabetic-free individuals in the first wave, estimates the following model

$$
\iota=\iota_{0} \exp (\tau Z)
$$

where $\iota$ is the instantaneous risk of T2D, $\iota_{0}$ is the risk of T2D among normal weight individuals, $\exp (\tau)$ is the relative risk of $\mathrm{T} 2 \mathrm{D}$ among obese individuals and $\mathrm{Z}$ is a $1 / 0$ binary variable for obesity. 
Expressed in terms of parameters in the multistate model $\iota_{0} \exp (\delta)$ is an estimate of $\mu_{26}$ and $\iota_{0}$ an estimate of $\mu_{14}$. The estimates of this two state model cannot be affected by preceding debilitation since, if there is any, its cause could not possibly be the event being studied, e.g., T2D experienced in the interwave interval cannot be the cause of weight loss prior to the first wave. ${ }^{11}$ Nor can they be affected by in-panel debilitation as that would require that an ancillary illness be the direct cause of incident cases of T2D, a rather odd circumstance. However, estimates may be influenced by transitions involving obese and normal weight individuals who contract T2D and die of it before the second wave. In the multistate model these are paths with transition rates $\mu_{14} \mu_{47}$ and $\mu_{26} \mu_{67}$.

The conventional two-state estimator of $\exp (\tau)$ is

$$
\exp (\hat{\tau})=\left(T 2 D_{1} / N_{1}\right) /\left(T 2 D_{0} / N_{0}\right)
$$

where $\mathrm{T}_{2} \mathrm{D}_{0}$ and $\mathrm{T} 2 \mathrm{D}_{1}$ are the number of recorded new cases of $\mathrm{T} 2 \mathrm{D}$ among normal weight and obese individuals. The relation to the true parameters is

$$
\exp (\hat{\tau})=\left(\left(1-\mu_{67}\right) /\left(1-\mu_{47}\right)\right) \exp (\tau)
$$

where $\mu_{47}$ and $\mu_{67}$ are the transitions rates from states 4 to 7 and 6 to 7 in the multistate model or, equivalently, the mortality rates of those with T2D in the normal weight and obese population respectively. Thus, the direction of the bias can vary depending on the lethality of T2D in the obese and normal weight population. In the second stage we generate empirical estimates of both $\mu_{47}$ and $\mu_{47}$ and then use them to adjust $\exp (\hat{\tau})$ iteratively.

\subsubsection{Stage 2: two-state model for the influence of T2D on mortality}

In a second stage we pose a simple-two state model to assess effects of T2D on mortality. Although in theory the computation of total effects of obesity through T2D only requires knowledge of the effects of T2D on mortality in the obese population, we estimate a model in both sub-populations:

$$
\begin{aligned}
& \mu_{d 0}=\mu_{d 00} \exp \left(\sigma_{0} T\right) \\
& \mu_{d 1}=\mu_{d 01} \exp \left(\sigma_{1} T\right)
\end{aligned}
$$

The first equation is for the normal weight and the second for the obese sub-populations; $\mu_{d 0}$ and $\mu_{d 1}$ are the instantaneous mortality rates due to illnesses other than T2D; $\sigma_{0}$ and $\sigma_{0}$ are effects of $\mathrm{T} 2 \mathrm{D}$ in the normal weight obese populations respectively, and $\mathrm{T}$ is a $1 / 0$ variable for $\mathrm{T} 2 \mathrm{D}$ at the first wave. Note that in the multistate model $\mu_{d 0}$ and $\mu_{d 1}$ correspond to the transition rates $\mu_{67}$ and $\mu_{47}, \mu_{d 00}$ and $\mu_{d 01}$ represent the transition rates $\mu_{57}$ and $\mu_{37}$ whereas $\exp \left(\sigma_{0}\right)$ and $\exp \left(\sigma_{1}\right)$ refer to the associated relative risks. Only one situation may produce distorted estimates, namely, when individuals who are T2D-free in the first wave contract the disease in the interwave period and then die before the second wave. Due to lack of information in limited panels, the investigator cannot identify these cases. The consequence of these unobserved transitions will be to under estimate the relative risks of $\mathrm{T} 2 \mathrm{D}$ in both sub-populations. If we use the parameters of the multistate model to express the ML estimators we obtain:

$$
\left.\exp \left(\hat{\sigma}_{0}\right) \sim \exp \left(\sigma_{0}\right) /\left(1+\mu_{26}\left(\mu_{67} / \mu_{57}\right)\right)\right)
$$

\footnotetext{
${ }^{11}$ It is, of course, possible that before the first wave individuals lose weight due to diseases unrelated to T2D, contract $\mathrm{T} 2 \mathrm{D}$ during the interwave, and die as a consequence of it rather than of the original illness that triggered the loss of mass to begin with.
} 
and

$$
\left.\exp \left(\hat{\sigma}_{1}\right) \sim \exp \left(\sigma_{1}\right) /\left(1+\mu_{14}\left(\mu_{47} / \mu_{37}\right)\right)\right)
$$

In addition, we will also obtain estimates of the baseline hazards $\mu_{d 00}$ and $\mu_{d 01}$.

Together, expressions (3.6), (3.9) and (3.10) suggest an iterative adjustment procedure. The first stage described above produces (potentially downwardly biased) estimates of $\mu_{26}$ and $\mu_{14}$. If we use these in expressions (3.9) and (3.10) we will generate partially adjusted estimates of $\exp \left(\sigma_{0}\right)$ and $\exp \left(\sigma_{1}\right)$ as well as of $\mu_{d 00}$ and $\mu_{d 01}$. Jointly, these values define $\mu_{47}$ and $\mu_{67}$ which can then be used in expressions (3.5) and (3.6) to compute a new estimate of $\exp (\tau)$. One can repeat these operations in a second iteration until additional changes in the final estimates are arbitrarily small. The mechanics of the adjustment in each iteration should lead to convergence to a unique value which can be considered the best estimate available from the data.

\subsubsection{Stage 3: assessment of the magnitude of the effects of obesity operating through well-defined illnesses}

In the third stage one combines estimates of relative risks of T2D and relative risks of mortality due to T2D in the obese sub-population. Essentially one needs to compute two mortality patterns, one unaffected by obesity-related conditions and one influenced by obesity-related conditions. We start with a baseline cohort-specific mortality pattern which is then altered to account for the impact of obesity on prevalence of T2D and of this on mortality. This can be done using extant forecasts of age patterns of obesity prevalence that are then combined with cohort-specific estimates of accumulated T2D and T2D-related mortality. The result will be forecasts of cohort-specific mortality patterns influenced by obesity (only via T2D). Finally, we compare forecasts with and without the influence of obesity.

\section{Illustration of the three stage procedure: the case of Mexican older adults}

We now illustrate the application of the three-stage procedure and estimate effects of obesity on mortality in a cohort of older Mexicans. We choose Mexico because it is a large middle income country with one of the highest obesity prevalence in the world and with a rich source of information. We focus on T2D as the target mediating chronic illness that produces excess mortality in the obese population. We first estimate a model for the relation between T2D and obesity and then a second model for the relation between T2D and mortality. We then combine these estimates and generate total effects of obesity on the force of mortality that operate via T2D. Finally, we estimate the number of years of life lost after age 60 implied by the the impact of obesity via T2D only.

\subsection{Data}

We use three waves of the Mexican Health and Aging Survey (MHAS) sweeping the period 20002012 (http://www.mhasweb.org). Target sample individuals (and spouses) were interviewed in the first wave (2001) and followed through a second (2002) and third (2012) waves. The baseline sample consists of 15,402 interviews carried out during 2001. Respondents were selected following the National Employment Study sampling protocol, conducted by the Instituto Nacional de Estadistica, Geografia e Informatica (INEGI), and covered both urban and rural residents in all 32 Mexican states. States with high emigration rates to the U.S. were over-sampled. Interviews were conducted in-person by INEGI professional interviewers and field supervisors administered a number of fitness tests and retrieved anthropometric measures, including height, weight, knee height, hip and waist 
circumference, to a $20 \%$ random sub-sample. Two follow-up interviews with surviving respondents were conducted during 2003 and 2012. The second wave consists of 14,386 respondents and a new sample of 220 new spouses. The third wave is made up of 12,569 respondents and a new, refreshment, sample of 5,896 new subjects and spouses. Next-of-kin proxy respondents reported 546 deceased respondents from the 2001 baseline and 2,742 deceased from the 2003 wave. In the application below we use the sample of targets which includes individuals of all marital status. The total size of this sample in the first wave is 9,609 . The total number of reported T2D in the first wave is 1,491 and the number of incident cases over the entire period is 895. Altogether this sample experienced a total of 2,265 deaths. We retrieve information on dates of deaths, self-reported health conditions, anthropometry (height and weight) as well as as self-reported height and weight.

\subsection{Stage 1: estimates of effects of obesity on T2D}

MHAS does not include information of the exact date of onset of T2D. As a consequence we cannot estimate a hazard model to estimate the impact of obesity on T2D. However, as shown in section 2 of the Appendix, we can transform the regression coefficients of a simple logit model for T2D into approximations of parameters of a hazard model.These are then used in the remaining computations. The logit model includes controls for gender and the log of age (see section 2 of the Appendix). Obesity is included as a set of three dummies for underweight, overweight and obese with normal weight used as the omitted category. Table 1 (panels A and B) displays estimates of the parameters of the logistic models and of predicted values of the corresponding hazards and relative risks respectively. The effects of the variable of interest are strong, statistically significant and in the expected direction. Further, the effects are graded so that the integrated risk of T2D over the 11-12 year period amounts to .056 among normal weight individuals, .13 among the overweight and .15 among the obese with relative risks of 1.18 and 2.67 respectively.

\subsection{Stage 2: Effects of T2D on mortality}

Table 2 contains estimates of four variants of parametric (Gompertz) hazard models. The first twostate model includes controls for age and gender as well as the dummies for obesity. The second two-state model adds a control for smoking. The third and fourth columns display estimates computed in the obese and normal weight sub-populations. They also include controls for age and gender and a dummy for T2D in the first wave. The mortality effects of T2D are very large and similar in the two sub-populations and correspond to relative risks in the range [1.97, 2.02]. This is a massive impact on the force of adult mortality.However, because the risks of contracting T2D in the normal weight sub-population is about half of the risk among the obese, the bulk of the impact of T2D on mortality is through obesity prevalence. We show this below where we chain together risks of contracting $\mathrm{T} 2 \mathrm{D}$ and $\mathrm{T} 2 \mathrm{D}$ specific mortality risks to produce estimates of the total impact of obesity.

If the researcher chooses a conventional two-state model to assess the total effects of obesity on mortality, she would privilege the results in columns 1 and 2. These models suggest that obesity has a beneficial impact on mortality: the relative risks of mortality are between 70 and 80 percent of the risk for normal weight individuals. The magnitude of the coefficients is not trivial and all are statistically significant, whether or not we control for smoking as a potentially confounding variable. 


\subsection{Combining results from stages 1 and 2}

To stitch together the estimates of the first two stages into a single estimate of the effect of obesity and mortality we follow a simple described in section 3 of the Appendix. The bottom line of the application is this: under conditions prevailing in Mexico, where the prevalence of obesity among adults older than 60 increased from about .30 to .60 in the span of thirty years (1985-2015), we would expect that mortality over age 60 should be subject to an upward pressure equivalent to losses in life expectancy at age 60 of about .8-1.0 years or between 5 and 10 percent of expected value of life expectancy at age 60 in 2020 . These losses of life are associated with only one chronic illness, T2D, and do not account for impacts of other obesity-related chronic diseases. Also, these are all virtual losses since mortality due to causes unrelated to obesity may continue to improve in the near future and thus offset negative effects of T2D. The estimated losses are better understood as the magnitude of a latent force, the strength of a grinding resistance working against additional mortality improvements.

\section{Discussion}

There is a large body of research on the effects of obesity on mortality. Although findings usually vary, and sometimes significantly so, it is thought that obesity, but possibly not overweight, produce excess adult mortality at adult ages. The findings and magnitudes involved, however, are not consistent across studies and in some cases obesity appears to have beneficial effects, particularly at very old ages. In this paper we show that (a) conventional estimates of obesity effects on mortality do not, as a norm, have a clear interpretation; (b) under some conditions routinely found in studies with limited panels the two-state estimates are biased; and (c) in all cases the comparability of estimates may be severely compromised since the two-state estimates conflate processes that could be very different in the populations being compared.

Precise estimates of obesity's total mortality effects require longitudinal studies and a multistate hazard approach. But estimation of a multistate model is not possible with limited panel designs of the sort we routinely have access to. A second best strategy is to use a three-stage estimation approach that, while not solving all problems, at least yields interpretable and comparable estimates under most conditions generating limited panel data.

There are a number of directions that research in this area can be extended to. First, one could fine-tune the iterative procedure employed in the three-stage approach, provide convergence conditions for the proposed algorithm, and derive properties of the estimators.

Second, one should use data from rich longitudinal studies to assess distortions associated with parameter estimates from a two-state model. Since the data from a detailed longitudinal study can be reduced to observable data that would have been produced by a limited panel, estimates from both a multistate and a two-state model can be generated and compared. Differences between estimates from the full and the (pseudo) limited panels should offer clues about the origins of biases, inconsistencies and ambiguities as well as about potential tools to avoid them.

Finally, further light must be shed on comparability problems of two-state estimates for subpopulations or countries where conditions regulating the incidence and fatality rates of illnesses associated with obesity are heterogeneous. This is particularly relevant in light of the increasing importance and influence of results from meta-analyses that employ a large number of studies based on highly diverse data sets, many of which are subject to pitfalls identified here. 
Table 1: Table 1: Logit model for incidence of T2D

\begin{tabular}{lcc}
\hline & \multicolumn{2}{c}{ A. Estimates from Logit Model (a) } \\
\hline Variable & Estimate & SE \\
& & \\
Log Age & -0.335 & 0.043 \\
Gender (1 males) & -0.203 & 0.055 \\
Underweight & -1.18 & 0.72 \\
Overweight & 0.593 & 0.12 \\
Obese & 1.05 & 0.12 \\
& & \\
Constant & -1.56 & 0.15 \\
N & 5472 \\
Log Likelihood & -1550.1 \\
Pseudo R-square & 0.0485
\end{tabular}

B. Estimated functions (from logit model)(b)

Cumulated probability of contracting T2D Yearly incidence rate of T2D

$\begin{array}{lcc}\text { Normal weight } & 0.056 & 0.005 \\ \text { Overweight } & 0.13 & 0.011 \\ \text { Obese } & 0.15 & 0.013\end{array}$

(a) Excludes observations with pre-existing T2D

(b) All quantities compute assuming age $=60$, gender $=1$ (males) 


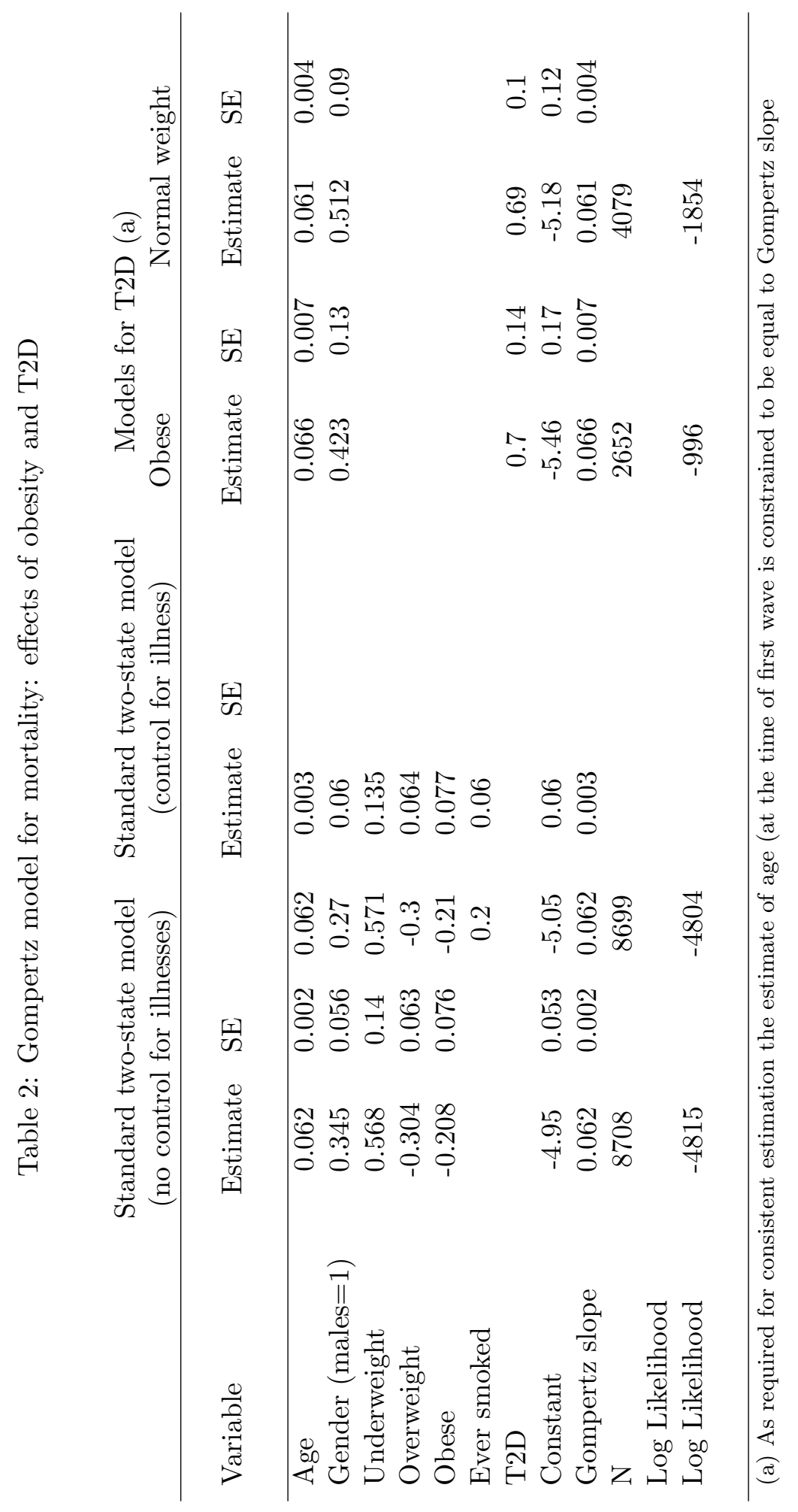




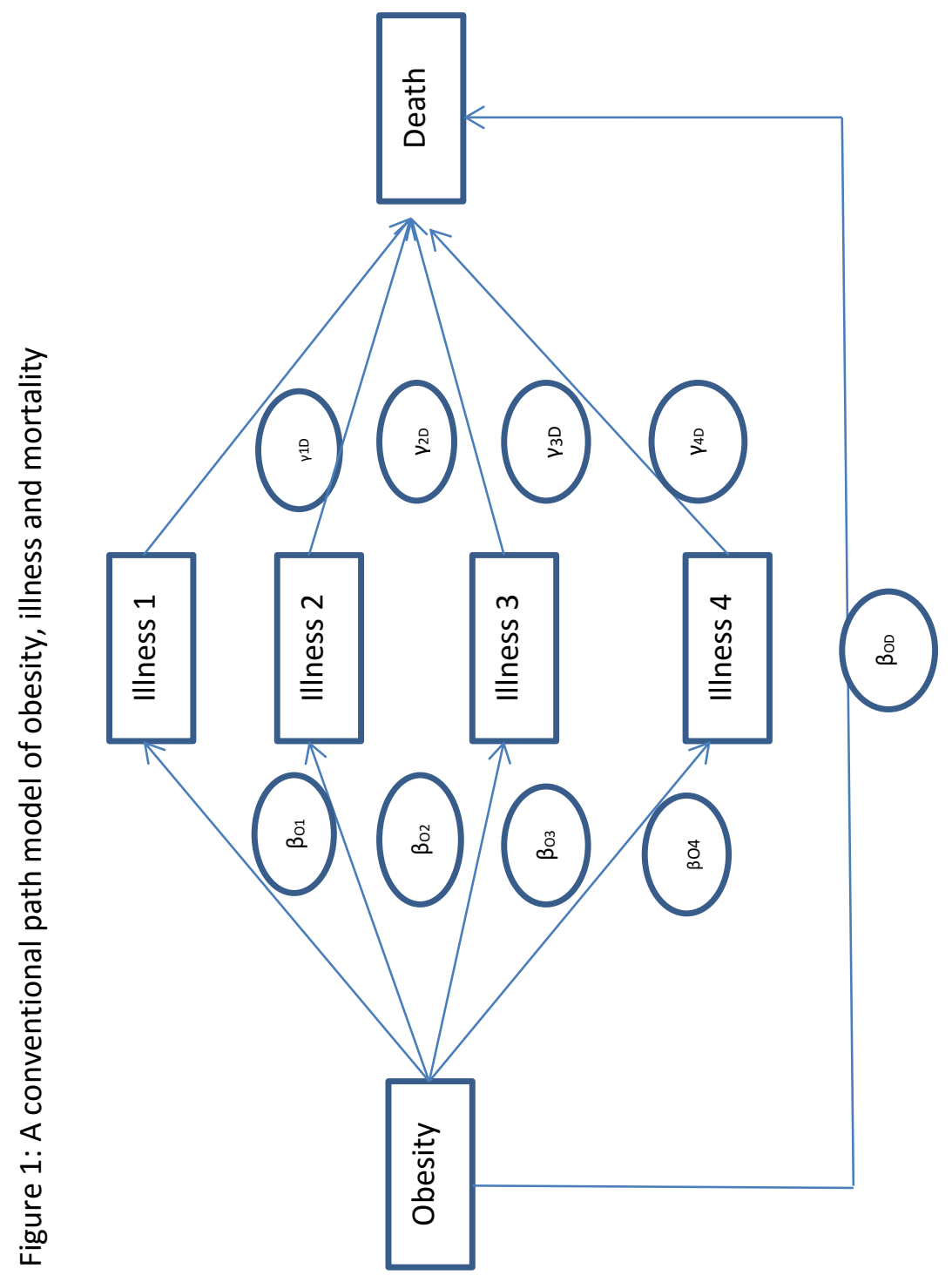




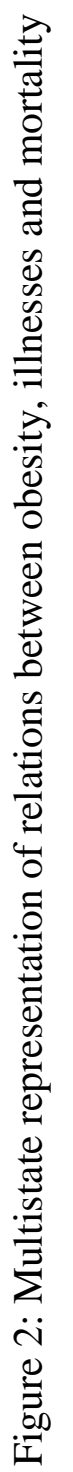

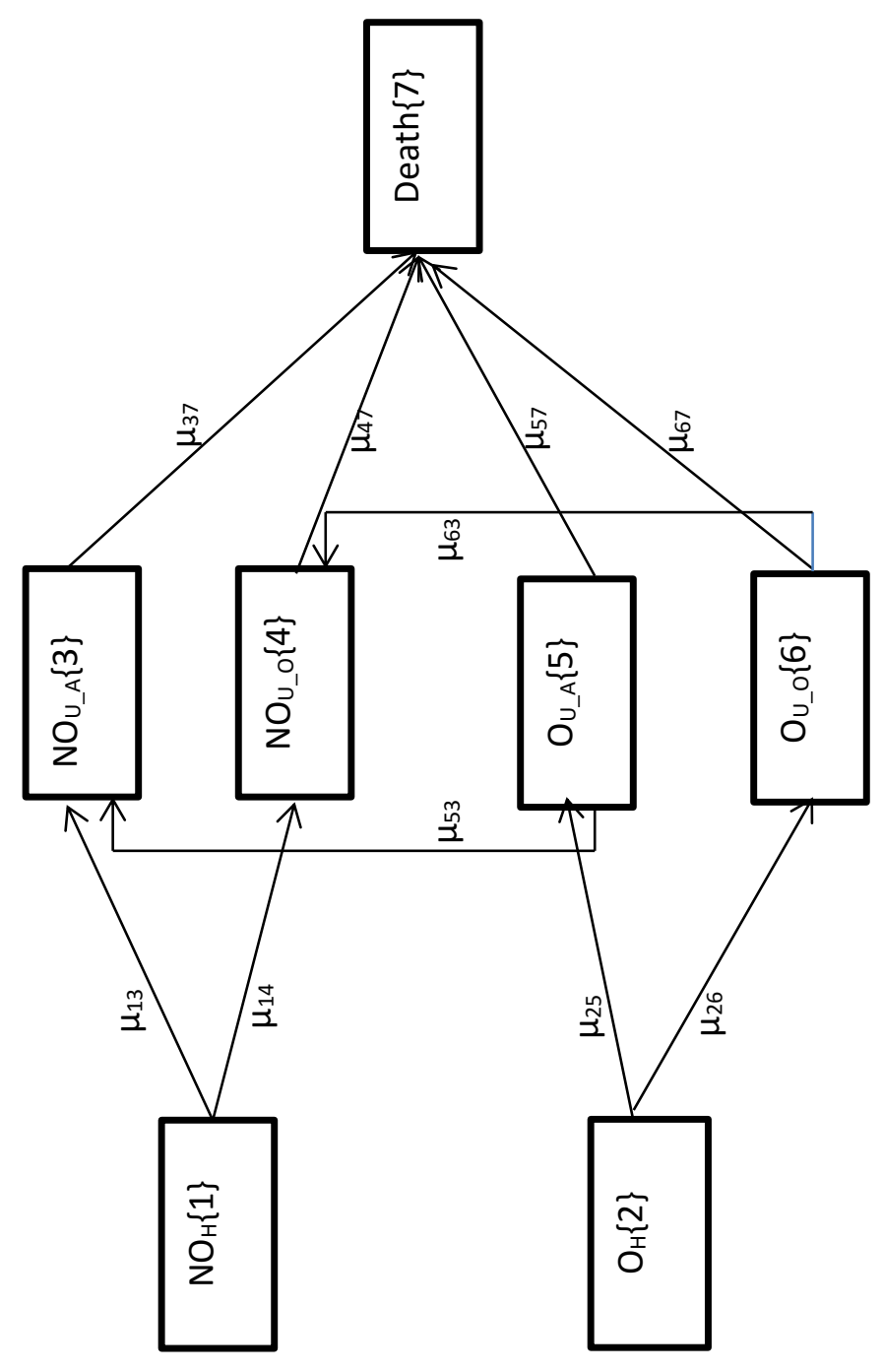




\title{
APPENDIX. The Impact of Obesity on Adult Mortality: Assessment of Estimates with Applications
}

\author{
Alberto Palloni* \\ and \\ Hiram Beltrán-Sánchez ${ }^{\dagger}$
}

\section{Derivation of expressions for reduced form of parameters in the two-state hazard model}

We proceed to derive expressions of the two-state parameters for cases with and without preceding debilitation. Throughout we use the multistate model specification set forth in section 2.3.

\subsection{Case I: No preceding debilitation $\left(\mu_{53}=\mu_{64}=0\right)$}

The ML estimates of the two parameters in the two-state hazard model correspond to the mortality rate in the left out category (non obese) and the ratio of the mortality rate in the obese population to the one in the non-obese population. We seek expressions of these two quantities using the relations embedded in the multistate hazard model. The observed number of deaths in the two sub-populations are:

$$
\begin{array}{r}
D_{n o}=N_{n o}\left[\operatorname { e x p } \left(-(\varepsilon / 2)\left(\mu_{13}+\mu_{14}\right) \mu_{13} \exp \left(-(\varepsilon / 4) \mu_{37}\right) \mu_{37}+\right.\right. \\
+\exp \left(-(\varepsilon / 2)\left(\mu_{13}+\mu_{14}\right) \mu_{14} \exp \left(-(\varepsilon / 4) \mu_{47}\right) \mu_{437}\right]
\end{array}
$$

or

$$
D_{n o}=N_{n o} S_{n o}\left(\mu_{13} \mu_{37}+\mu_{14} \mu_{47}\right)
$$

where $D_{n o}$ and $N_{n o}$ are the numbers of deaths and subjects exposed at the outset, respectively, in the non obese population. The function $\mathrm{S}_{n o}$ is shorthand for $\exp \left(-(\varepsilon / 2)\left(\mu_{13}+\mu_{14}\right)\right.$. We assumed that the total interval of exposure is $\varepsilon$ and that the first and second events take place in the middle of the interval of exposure. When risks and intervals of exposure are small we can use the approximation $\exp (-(\varepsilon / 4) \mu) \sim 1$ ) and obtain (1.2) from (1.1). A similar expression holds for the observed number of deaths in the obese population

$$
D_{o}=N_{o} S_{o}\left(\exp (\lambda+\phi) \mu_{13} \mu_{37}+\exp (\delta+\beta) \mu_{14} \mu_{47}\right)
$$

where we use the multistate hazard specification relating risks in the two sub-populations (see section 2.3). Because the total exposure (denominator of the rates) will be $\varepsilon N_{n o} S_{n o}$ and $\varepsilon N_{o} S_{o}$

\footnotetext{
${ }^{*}$ Center for Demography of Health \& Aging, University of Wisconsin-Madison. Email: palloni@ssc.wisc.edu.

${ }^{\dagger}$ Department of Community Health Sciences at the Fielding School of Public Health \& California Center for Population Research, UCLA. Email: beltrans@ucla.edu.
} 
respectively, the ratio of death rates, that is, the ML estimate of the effects of obesity on mortality, will be the quantity

$$
\hat{\rho_{I}}=D_{o} / D_{n o}=w \exp (\lambda+\phi)+(1-w) \exp (\delta+\beta)
$$

where $w=\mu_{13} \mu_{37} /\left(\mu_{13} \mu_{37}+\mu_{14} \mu_{47}\right)$.Expression (1.4) shows the contribution to the two-state estimate of transition rates involving both obesity related and ancillary conditions. If $\beta$ and $\delta$ are large relative to other parameters or if, irrespective of type of illness, the relative risks are the same across the two sub-populations, the two state estimate will reflect what we refer to as the total effect of obesity or the value of $\exp (\delta+\beta)$, that is the "product" of a path leading from obesity to obesity-related illness and the path from obesity-related illness to death. If these conditions are not met the two-state estimate will always reflect the relative strength of multiple paths or mechanisms. Thus, two populations may yield similar two-state estimates of obesity even though the mechanisms leading to them could be quite different. Conversely, two populations may have the same values of $\delta$ and $\beta$ but yield different two-state estimates of the effect of obesity. For example, if in populations $\mathrm{A}$ and $\mathrm{B}$ the total effect of obesity is given by $\exp (\delta+\beta)$ but the transition rates from state 2 to 5 and/or from state 5 to 7 both involving non-obesity related deaths, are different, one would conclude that the total effects in A and B are different, when in fact they are the same. The difference could be explained by factors spuriously associated with obesity but not with any mechanism that leads from obesity to excess deaths.

\subsection{Case II: Preceding debilitation: $\mu_{53}$ and $\mu_{64}$ different from 0}

The introduction of preceding debilitation leads to reduced form expressions that contain additional transition rates that produce biases and complicate even more the interpretation of the two-state total estimated effects of obesity. The bias is a result of the fact that the quantity $D_{o}$ now includes deaths to obese individuals who experience debilitation and move between states 5 or 6 to state 3 and then to state 7 . We invoke the same assumptions we used before and express the ratio of observed death rates in the two sub-populations of interest in terms of the parameters of the multistate model. The number of deaths in each sub-population is given by

$$
D_{n o}=N_{n o} S_{n o}\left(\mu_{13} \mu_{37}+\mu_{14} \mu_{47}\right)+N_{o} S_{o}\left(\mu_{25} \mu_{53} \hat{\mu}_{37}+\mu_{26} \mu_{64} \hat{\mu}_{47}\right)
$$

and

$$
D_{o}=N_{o} S_{o}\left(\mu_{25} \mu_{57}+\mu_{26} \mu_{67}\right)
$$

Using the parameters of the multistate models leads to an expression for the ratio of the two death rates

$$
\begin{array}{r}
\hat{\rho}_{I I}=\left\{\mu_{13} \mu_{37} \exp (\lambda+\phi)+\mu_{14} \mu_{47} \exp (\delta+\beta)\right\} /\left\{s_{o}\left(\mu_{13} \mu_{37}+\mu_{14} \mu_{47}\right)+\right. \\
\left.+(1-s)\left(\mu_{15} \mu_{53} \mu_{37} \exp (\delta+\beta)+\mu_{14} \mu_{64} \mu_{47} \exp (\delta+\varphi)\right)\right\}
\end{array}
$$

where $s$ is the ratio of the exposure contributed by those who transit to state 3 from states 5 or 6 to the total exposure in the non-obese population. If we assume that these two sub-populations were exposed the same amount of time, we can express (1.7) as

$$
\hat{\rho}_{I I}=\hat{\rho}_{I}\left\{1+\mu_{13} \mu_{37} \mu_{53} \exp (\alpha+\gamma)+\mu_{14} \mu_{47} \mu_{64} \exp (\delta+\varphi)\right\}^{-1}
$$


a product of the estimate obtained when there is no preceding debilitation and a quantity that will always be lower than 1 . It has all the interpretational ambiguities of $\hat{\rho}_{I}$ and, in addition, it contains a systematic downward bias.

\section{The parameters of a logistic model for T2D incidence to ap- proximate $\mu_{14}$ and $\mu_{26}$}

The logistic model for T2D incidence we used is :

$$
\ln (Q(x) /(1-Q(x))=\alpha+\beta Z+\gamma g(x)
$$

where $Q(x)$ is the probability of contracting T2D among individuals aged $x$ in the first wave during the time interval elapsed between the first and last wave, $Z$ is a vector of covariates, and $g(x)$ some function of age. Also $Q(x, t)=(1-\exp (-I(x))$ where $I(x)$ is the integrated risk of contracting T2D among individuals aged $x$. In the case we study $I(x)$ does not exceed .15 and we can express the logit of $Q(x)$ as ${ }^{1}$

$$
\ln (Q(x) /(1-Q(x))=\ln (\exp (I(x)-1) \sim \ln I(x)
$$

so that

$$
I(x) \sim H(x) \exp (\beta Z)
$$

where $H(x)=\exp (\alpha+\gamma g(x))$ is a baseline integrated hazard evaluated at age $x$. If the rate of incidence is age invariant, $H(x)=\exp (\alpha)$. Thus, one can convert the parameters of a logit model for the (accumulated) incidence of T2D into parameters of a proportional hazard model for the integrated risk of T2D. From the predicted values of $I(x)$ we can compute discrete values of the risk of $\mathrm{T} 2 \mathrm{D}, \mu_{14}$ and $\mu_{26}$, in the non-obese and obese population respectively.

\section{Estimation of total effects of obesity on adult mortality and life expectancy}

To convert estimates from the two-stage procedure into quantitative measures of the influence of obesity on adult mortality we first compute effects on average mortality and then convert these into effects on life expectancy at age 60 .

\subsection{Effects on adult mortality}

Assume we follow cohorts who attain age 60 during the period 2000-2012. This population is composed of obese and normal weight individuals some of whom contracted T2D earlier on in their lives. The average mortality rate at age 60 is $\bar{\mu}=\mu_{n d}\left(\left(1-P_{d}\right)+\lambda P_{d}\right)$, where $P_{d}$ is the cohort's T2D prevalence, $\mu_{n d}$ is the mortality risk for non-diabetics and $\lambda$ is the relative risk of death among diabetics. ${ }^{2}$ Taking derivatives with respect to $P_{d}$ and rearranging we obtain

$$
\partial \bar{\mu} / \bar{\mu} \sim\left(\mu_{n d} / \bar{\mu}\right)(\lambda-1) P_{d}\left(\partial P_{d} / P_{d}\right)
$$

and the elasticity of the average mortality relative to T2D prevalence is

\footnotetext{
${ }^{1}$ When $I(x, t) \leq .15$, the approximation will be in error by less than $5 \%$

${ }^{2}$ To avoid cluttering we omit age and year subscripts.
} 


$$
\epsilon_{1}=\left(\mu_{n d} / \bar{\mu}\right)(\lambda-1) P_{d}
$$

Using MHAS we estimate that at age $60 P_{d}=.11$ and the ratio $\left(\mu_{n d} / \bar{\mu}\right)$ is approximately .50 . Our estimate from the logit models suggest that $\lambda$ is close to 2 . Thus,$\epsilon_{1}$ is in the neighborhood of .10, that is, a one percentage point increase in $\mathrm{T} 2 \mathrm{D}$ prevalence induces a .10 percent increase in the force of mortality at age 60 .

Following an analogous derivation we find the elasticity of $P_{d}$ relative to obesity prevalence

$$
\epsilon_{2}=\left(P_{d}^{n o} / P_{d}\right)(\varphi-1) O
$$

where $P_{d}^{n o}$ is the fraction of diabetics in the population of normal weight, $O$ is the prevalence of obesity, and $\varphi$ is the relative risk of T2D in the obese population. Using MHAS we estimate that at age 60 the ratio $\left(P_{d}^{n o} / P_{d}\right)$ is about .45 and from the estimated hazard model we know that $\varphi$ is close 3. The value of $\epsilon_{2}$ will be a function of $O$. We examine the role of this quantity below.

\subsection{Effects on life expectancy at age 60}

To illustrate the ultimate mortality effect of increasing obesity we assign two values to the prevalence of obesity $O$ in a cohort attaining age 60, namely, .60 (as in MHAS) and to an earlier cohort attaining age 60 during the 1980's, .30. The latter number is estimated as follow: first, we extrapolate backward to 1980 the linear trend of adult obesity observed during the period 1998-2012. Second, we assume that the ratio of older adult obesity to adult obesity observed in the most recent period has been constant throughout ${ }^{3}$

This doubling of adult obesity prevalence prevalence during these twenty or so years translates into an increase in mortality due to $\mathrm{T} 2 \mathrm{D}$ of the order of 6 percent $\left(100^{*} \epsilon_{1}{ }^{*} \epsilon_{2}\right)$. If this shift in mortality rates applies to all ages over 60 , the upward trend in obesity prevalence since the early eighties would lead to a decrease in life expectancy of about 3 percent. This proportionate loss in life expectancy at age $60, \Delta E_{60}$, is computed as $\Delta E_{60} \sim \delta \Gamma_{60}$, where $\delta$ is the age invariant proportionate change in the force of mortality and $\Gamma_{60}$ is the entropy of the survival curve in a life table with $\mathrm{E}_{60}$ of about 18 years, the value most appropriate for Mexican males during the period we examine here. ${ }^{4}$ Mexican male life expectancy at age 60 during the 1980's was of the order of 18.3 and, by the year 2020, is expected to increase to about 22.19 , a 21 percent gain ${ }^{5}$. Thus, if there had been no excess mortality due to obesity via T2D the gain would have been 25 percent, for a life expectancy at age 60 in 2020 of about 23 years. To the extent that mortality due to other causes improves, these counterfactual losses will not be observed. Instead, they will simply impede additional gains in survival.

\footnotetext{
${ }^{3}$ See Cecchini and Sassi, 2014

${ }^{4}$ (See Keyfitz, N.1977 Applied Mathematical Demography, Wiley, New York, USA. We hasten to add that we use these approximations to facilitate the description of the illustration. In real life one could use standard and exact, if tedious, demographic techniques.

${ }^{5}$ Computed from https://esa.un.org/unpd/wpp/DataQuery/ as accessed in April 4th, 2017
} 


\section{References}

Al Snih, S., Ottenbacher, K. J., Markides, K. S., Kuo, Y.-F., Eschbach, K., and Goodwin, J. S. (2007), "The effect of obesity on disability vs mortality in older Americans," Archives of internal medicine, 167, 774-780.

Biro, F. M. and Wien, M. (2010), "Childhood obesity and adult morbidities," The American journal of clinical nutrition, 91, 1499S-1505S.

Brisbois, T., Farmer, A., and McCargar, L. (2012), "Early markers of adult obesity: a review," obesity reviews, 13, 347-367.

Cecchini, M. and Sassi, F. (2014), "Obesity Update," Tech. rep., OECD (www.oecd.org/health/prevention).

FAO (2015), "The state of food insecurity in the world," http://www.fao.org/hunger/en/(accessed on $4 / 4 / 201 \%$.

Flegal, K. M., Kit, B. K., Orpana, H., and Graubard, B. I. (2013), "Association of all-cause mortality with overweight and obesity using standard body mass index categories: a systematic review and meta-analysis," Jama, 309, 71-82.

Fontaine, K. R., Redden, D. T., Wang, C., Westfall, A. O., and Allison, D. B. (2003), "Years of life lost due to obesity," JAMA, 289, 187-193.

Guh, D. P., Zhang, W., Bansback, N., Amarsi, Z., Birmingham, C. L., and Anis, A. H. (2009), "The incidence of co-morbidities related to obesity and overweight: a systematic review and meta-analysis," BMC public health, $9,88$.

Ix, J. H. and Sharma, K. (2010), "Mechanisms linking obesity, chronic kidney disease, and fatty liver disease: the roles of fetuin-A, adiponectin, and AMPK," Journal of the American Society of Nephrology, 21, 406-412.

Ng, M., Fleming, T. andRobinson, M. T. B., and Graetz, N. e. a. (2014), "Global, regional, and national prevalence of overweight and obesity in children and aduts during 1980-2013: a systematic analysis for the Global Burden of Disease Study 2013," The Lancet, 384, 766-81.

Preston, S. H. and Stokes, A. (2011), "Contribution of obesity to international differences in life expectancy," American Journal of Public Health, 101, 2137-2143.

Preston, S. H., Stokes, A., Mehta, N. K., and Cao, B. (2014), "Projecting the effect of changes in smoking and obesity on future life expectancy in the United States," Demography, 51, 27-49. 